\title{
Deciphering the underlying mechanism of Xianlinggubao capsule against osteoporosis by network pharmacology
}

\author{
Hangsheng Bao ${ }^{1}$, Huizhi Guo ${ }^{2}$, Zongquan Feng ${ }^{1}$ and Xin $\mathrm{Li}^{3^{*}}$
}

\begin{abstract}
Background: Xianlinggubao formula (XLGB), a Chinese State Food and Drug Administration-permitted traditional Chinese herbal medicine, has been extensively used to treat osteoporosis. Although XLGB was shown to improve bone mass in ovariectomized rats and clinically alleviate osteoporosis symptoms, its pharmacological mechanisms remain unclear.
\end{abstract}

Methods: In this study, we used a network pharmacological approach to explore the potential mechanism of XLGB in treating osteoporosis. We obtained XLGB compounds from the TCMSP and TCMID databases and identified potential targets of these compounds through target fishing based on the TCMSP and Swiss Target Prediction databases. Next, we identified the osteoporosis targets by using the CTD, TTD, GeneCards, OMIM and PharmGKB databases. Then, the overlapping genes between the XLGB potential targets and the osteoporosis targets were used to establish a protein-protein interaction (PPI) network and to analyze their interactions and identify the major hub genes in this network. Subsequently, the Metascape database was utilized to conduct the enrichment of Gene Ontology biological processes and Kyoto Encyclopedia of Genes and Genomes (KEGG) pathways.

Results: There were 104 active compounds and 295 related targets identified overall. After the Metascape enrichment analysis, we identified the top 25 cellular biological processes and top 15 pathways based on the logP value and found that the XLGB-mediated anti-osteoporosis effect was mainly associated with reactive oxygen species, organonitrogen compound response and cell migration. Furthermore, 36 hub genes of XLGB, such as EGF, EGFR, MTOR, MAPK14 and NFKB1, were considered potential therapeutic targets, suggesting the underlying mechanisms of XLGB acting on osteoporosis.

Conclusion: We investigated the possible therapeutic mechanisms of XLGB from a systemic perspective. These key targets and pathways provide promising directions for future research to reveal the exact regulatory mechanisms of XLGB.

Keywords: Xianlinggubao capsule, Network pharmacology, Osteoporosis

\footnotetext{
* Correspondence: xinli20190719@163.com

${ }^{3}$ Department of Nephrology, The First Affiliated Hospital of Guangzhou

University of Chinese Medicine, Guangzhou 510405, China

Full list of author information is available at the end of the article
}

(c) The Author(s). 2020 Open Access This article is licensed under a Creative Commons Attribution 4.0 International License, which permits use, sharing, adaptation, distribution and reproduction in any medium or format, as long as you give appropriate credit to the original author(s) and the source, provide a link to the Creative Commons licence, and indicate if changes were made. The images or other third party material in this article are included in the article's Creative Commons licence, unless indicated otherwise in a credit line to the material. If material is not included in the article's Creative Commons licence and your intended use is not permitted by statutory regulation or exceeds the permitted use, you will need to obtain permission directly from the copyright holder. To view a copy of this licence, visit http://creativecommons.org/licenses/by/4.0/ The Creative Commons Public Domain Dedication waiver (http://creativecommons.org/publicdomain/zero/1.0/) applies to the data made available in this article, unless otherwise stated in a credit line to the data. 


\section{Background}

Osteoporosis (OP) is a systemic bone disorder characterized by low bone mass and an accompanying increased incidences of fractures. And OP is usually considered to be the result of an imbalance between osteoclasts promoting bone absorption and osteoblasts inducing bone remodeling [1]. A recent study noted that over 10 million people suffered from osteoporosis in the United States. Moreover, its treatment burden, $\$ 22$ billion in 2008 , is expected to rise due to the consistently increasing aging population. Related osteoporosis treatments should be used to alleviate or reduce symptoms such as fractures, which could result in substantial cost savings [2]. For inhibition of bone loss and maintenance of bone mass, antiresorptive agents, anabolic agents and bone mineral drugs have been widely used in clinical treatment [3]. However, notably, almost all these antiosteoporosis methods have limitations and side effects, such as increasing the risk of breast cancer, jaw necrosis or atypical femur fracture $[4,5]$. Therefore, the development of alternative and safe intervention strategies for osteoporosis is needed.

Traditional Chinese medicine (TCM) has attracted worldwide attention and served as a main alternative treatment in East Asia, North America, and Europe due to its satisfactory curative effect, relatively low toxicity and low cost [6-8]. TCM has been used in China for thousands of years and has been shown to have good therapeutic effects and few side effects through multiple herb combinations for the prevention and treatments of various diseases [9]. Furthermore, an increasing number of TCMs have been found to be effective in the treatment of osteoporosis $[10,11]$.

As an important type of TCM, Xianlinggubao formula (XLGB) has been proven to effectively improve bone mass in ovariectomized rats [12] and clinically alleviate osteoporosis symptoms [13]. A multicenter and double blind clinical trial confirmed its beneficial effect on postmenopausal women with osteoporosis as well [14]. XLGB, similar to other TCM formulas, consists of six herbs with percentages in weight: Epimedium sagittatum Maxim (70\%), Dipsacus inermis Wall. (10\%), Salvia miltiorrhiza Bunge (5\%), Anemarrhena asphodeloides Bunge (5\%), Psoralea corylifolia L. (5\%), and Rehmannia glutinosa Steud. (5\%) [12]. Due to its therapeutic efficacy and safety, the Chinese State Food and Drug Administration (CFDA) has officially permitted XLGB capsules to be sold as an overthe-counter (OTC) drug for osteoporosis treatment [14]. Although XLGB performed well in clinical practice, its exact therapeutic mechanism in osteoporosis is still unclear. Similar to all TCM decoctions, XLGB exerts its therapeutic efficacy by regulating multiple molecules in the human body. Therefore, it is still a major challenge to identify its mechanism through scientific trials that are used in Western medicine. Fortunately, the development of systems pharmacology offers researchers an alternative opportunity and option to investigate the pharmacological mechanisms of TCM [15]. Recently, Wang et al. utilized a network pharmacology method to clarify the synergistic mechanism of Er-Xian decoction in treating osteoporosis [16]. Similarly, Zhang et al. employed this systems pharmacology method to dissect the mechanisms underlying the therapeutic effect of Dipsacus inermis Wall. on osteoporosis [17].

In this network pharmacology study, we aimed to comprehensively dissect the mechanisms of XLGB in treating osteoporosis. We identified compounds related to the XLGB capsules based on multiple databases and obtained the compounds' potential targets via target fishing. Then, we matched them with osteoporosis-related targets collected through a multisource database. Next, using overlapping targets from the previous process, we built a protein-protein network to analyze their internal interactions and then identified the hub genes. Furthermore, we used the Metascape database to conduct the biological process and Kyoto Encyclopedia of Genes and Genomes (KEGG) pathway enrichment analyses. The protocol of our experimental procedures is shown in Fig. 1.

\section{Methods \\ Identification of chemical ingredients}

To establish a compound ingredient database, we utilized the Traditional Chinese Medicine System Pharmacology Database (TCMSP ${ }^{\mathrm{Tx}}$, http://lsp.nwu.edu. cn/tcmsp.php) [18], which is a frequently used platform for systems pharmacology. The Traditional Chinese Medicines Integrated Database (TCMID, http://119.3.41.228:8000/) [19] is one of the largest comprehensive TCM platforms. In total, one thousand and seventy-four chemical ingredients were identified in this part.

\section{Screening of active ingredients $O B$ evaluation}

Oral bioavailability (OB) refers to the percent of an orally administered drug that reaches systemic circulation and is one of the most important pharmacokinetic indexes for drug screening. The TCMSP platform has adopted the OBioavail1.1 system, which integrates P450, 3A4 and P-glycoprotein information to obtain the $\mathrm{OB}$ value [20]. To determine the active ingredients used for further steps, we set the $\mathrm{OB}$ threshold at $30 \%$. 


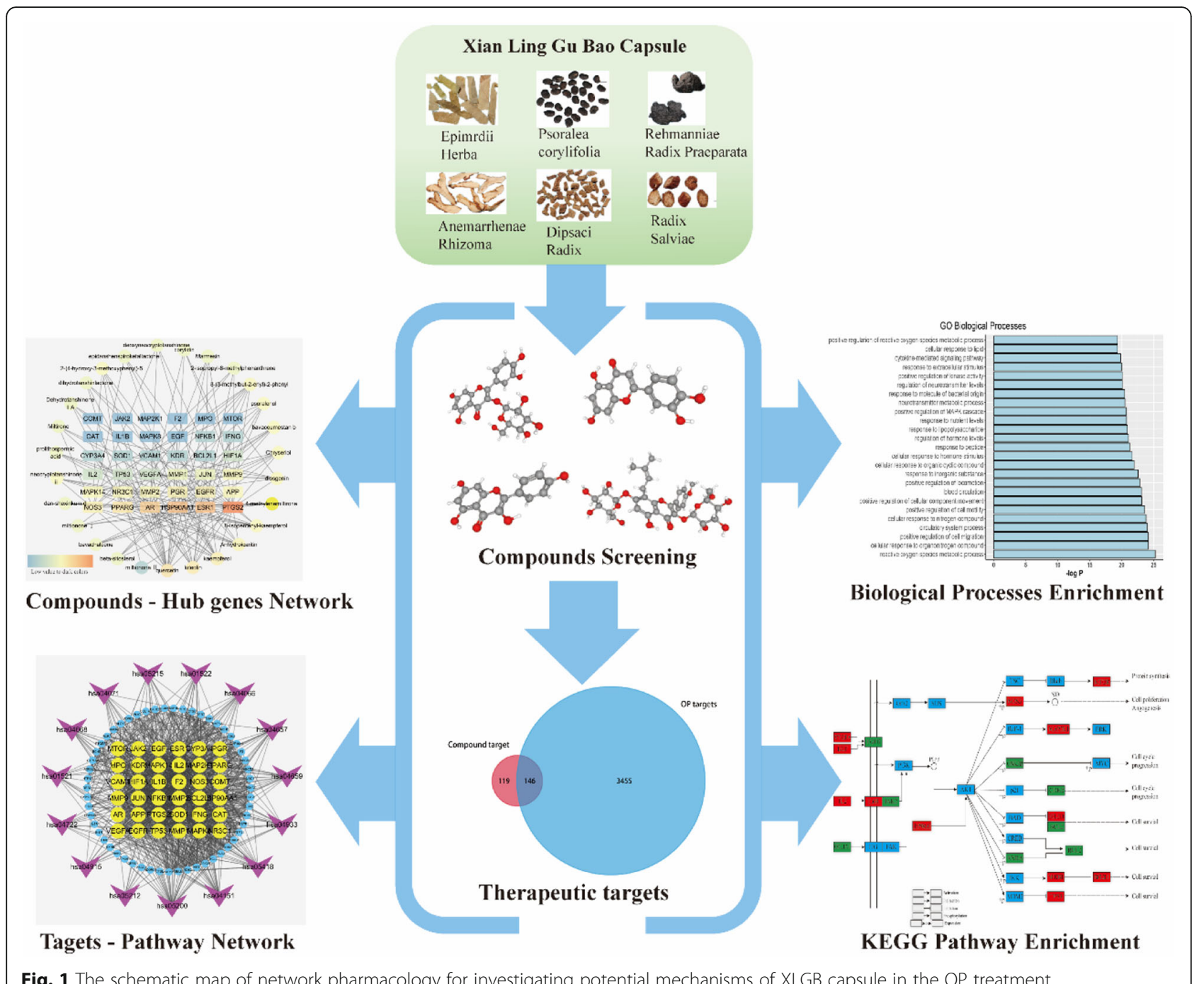

\section{DL prediction}

Drug-likeness (DL) is a qualitative index that represents the "drug-like" degree of the target compound and is used to remove chemically unsuitable compounds. TCMSP used the Tanimoto similarity method to calculate the DL index by comparing the target compound to all 6511 molecules in the DrugBank database [21]. In this process, the compounds that did not meet the condition that $\mathrm{DL} \geq 0.18$ were removed. For herbs that were not included in TCMSP, we used the Swiss ADME database (http://www.swissadme.ch/) [22] to conduct the screening process. This platform allowed us to compute the pharmacokinetic properties and drug-like nature of the target compounds. This database has five different rule-based filters to define how drug-like the compound is. All these filters, including Lipinski, Ghose, Veber, Egan and Muegge, are from major pharmaceutical companies aiming to improve the quality of their drug development [22]. Thus, the compounds that met at least four rules were considered active ingredients and used for the next steps.

\section{Caco-2 permeability}

The permeability of the target drug is another critical part of drug development. The participation of villi and microvilli strongly increases the small intestinal absorption of orally administered drugs. Thus, the TCMSP platform used the Caco-2 permeability model based on 100 drug molecules with satisfactory statistical results $(\mathrm{R} 2>0.8)$ to find compounds with good permeability. Therefore, we set the Caco-2 threshold at 0 , and the compounds that met these criteria were used for further experiments.

\section{Target prediction of active ingredients}

The compound ingredients have effects on the targets to exert their biological function. Thus, we used the TCMSP platform to predict the targets of active 
ingredients. For herbs that were not included in TCMSP, we used the Swiss Target Prediction database instead (http://www.swisstargetprediction.ch/index.php). After obtaining canonical descriptors of ingredients, we assessed their potential targets via this webserver based on chemical similarity.

\section{Disease target identification by multiple databases}

We used multiple databases to collect OP-related targets, and the term 'Osteoporosis' was searched as the key word. The databases in this step included the Comparative Toxicogenomics Database (CTD, http://ctdbase. org/) [23], GeneCards (https://www.genecards.org/) [24], the Therapeutic Target Database (TTD, https://db.idrblab.org/ttd/) [25], OMIM (https://www.omim.org/) [26] and PharmGKB (https://www.pharmgkb.org/) [27]. In total, three thousand six hundred and one OP target were found through these databases.

Then, we constructed a Venn diagram to determine the overlapping targets between the OP and active ingredient targets because these overlapping targets play a critical role when XLGB treats OP. These targets were analyzed by String (https://string-db.org/) [28], and the protein-protein interaction (PPI) data were exported.

\section{Network construction \\ Construction method}

Three main networks were built in this process, including 1. The compound-target network, 2. The XLGB target-OP target interaction network and 3 . The targetpathway network. Target information was obtained from the KEGG pathway enrichment results. Cytoscape 3.6.2 (http://www.cytoscape.org/) was used in all network visualizations and is one of most powerful open-source software programs for constructing different networks visually [29]. However, there are several limitations in this network pharmacology study, as we did not determine whether the compounds activate or inhibit the targets, how they mediate the effect on the targets, binding or catalysis, and so on.

\section{Topological feature definition}

We used three parameters to describe and quantify the importance of nodes in these networks because the nodes that bridge many edges with neighborhoods are more likely to exert crucial mediating functions. 1. 'Degree' indicates the number of edges with other nodes. Examining the node's degrees is the most straightforward method of quantifying the node centrality [30]. 2 . "Betweenness centrality" is used to measure code centrality based on the shortest paths. A high value node would play a more important role in the network because more regulation information will pass through it [31]. 3. "Closeness centrality" represents the mean distance between the node and all other nodes in the network and is the reciprocal of the sum of the length of the shortest paths between itself and all other nodes in the network. Therefore, the central node would be more likely to be close to all other nodes [32].

\section{Biological process and pathway enrichment analysis}

We used the Metascape database (http://metascape.org/ gp/index.html) [33] to conduct the Gene Ontology (GO) biological process enrichment analysis and conducted it based on Kyoto Encyclopedia of Genes and Genomes [34] (KEGG, http://www.kegg.jp/) data obtained from Metascape.

\section{Results}

\section{Active compounds of the XLGB capsules}

From the TCMSP and TCMID databases, we identified 553 related compounds in the formula, among which there were 130 ingredients $(23.5 \%)$ in Yin Yang Huo (Epimedium sagittatum Maxim, YYH), 202 (36.5\%) in Dan Shen (Salvia miltiorrhiza Bunge, DS), 76 (13.7\%) in Di Huang (Rehmannia glutinosa Steud, DH), 31 (5.6\%) in (Dipsacus inermis Wall., XD), 81 (14.6\%) in Zhi Mu (Anemarrhena asphodeloides Bunge, ZM), and 33 (6.0\%) in $\mathrm{Bu} \mathrm{Gu}$ Zhi (Psoralea corylifolia L., BGZ). After screening by the ADME thresholds $(\mathrm{OB} \geq 30 \%, \mathrm{DL} \geq 0.18$ and Caco-2 $>0$ ) and five different rule-based filters of the Swiss ADME database, we obtained 104 active compounds. Then, the herb-compound network was constructed as shown in Fig. 2. Subsequently, we analyzed and reordered these compounds in descending order of edge betweenness, and the top three compounds were sitosterol $(\mathrm{OB}=36.94, \mathrm{DL}=0.75$, Caco-2 $=1.32)$, luteolin $(\mathrm{OB}=36.16, \mathrm{DL}=0.25, \mathrm{Caco}-2=0.19)$ and stigmasterol $(\mathrm{OB}=43.83, \mathrm{DL}=0.76$, Caco- $2=1.44)$.

\section{Target prediction and analysis}

Next, we conducted target fishing for these 104 active ingredients using the TCMSP and Swiss Target Prediction databases based on chemical similarity. We obtained 295 related targets, among which BGZ had 191 targets, DS had 67 targets, DH had 27 targets, XD had 38 targets, YYH had 110 targets and ZM had 79 targets. We integrated the OP genes obtained from multisource databases, including CTD, GeneCards, TTD, OMIM and PharmGKB, and a total of 3601 related genes were identified After the construction of the Venn diagram, one hundred forty-six overlapping targets between the related targets of XLGB and OP were selected as the key targets through which XLGB exerts an anti-OP effect (Fig. 3).

The data obtained from the String database were used to establish the PPI network for the 146 overlapping targets. In this network, there were 146 nodes and 1522 edges in total. Then, three main parameters, "degree", 


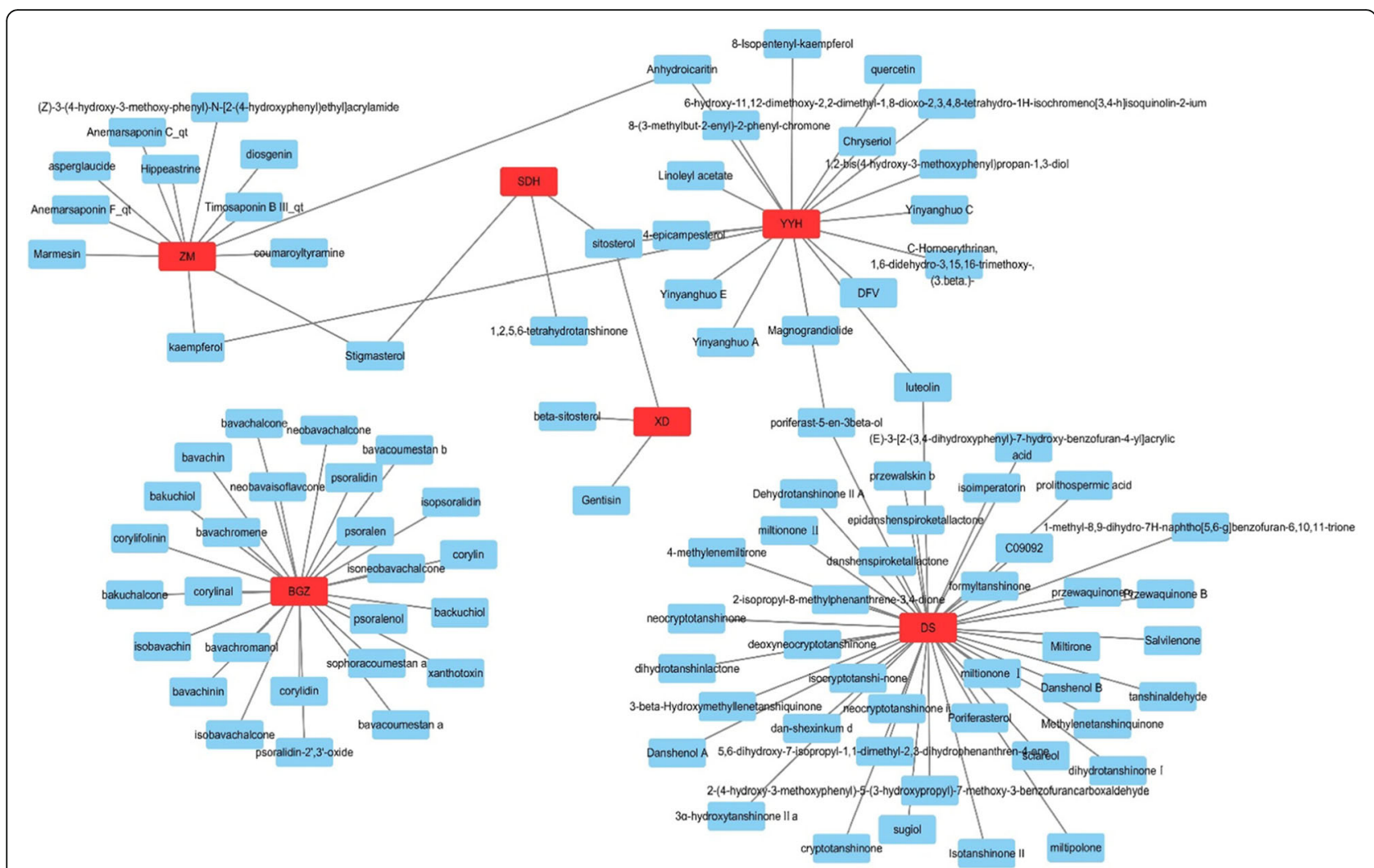

Fig. 2 The network of Herbs-Compounds connection. The red nodes represent ingredients in XLGB capsule while the blue ones represent active compounds

"betweenness", and "closeness", were used as filters to select the key genes and build up the major hub nodes for the anti-OP effect of XLGB. The first screening threshold was degree $\geq 17$, closeness $\geq 0.459$ and betweenness $\geq 0.002$, which resulted in 72 nodes and 942 edges. Then, these 72 key nodes were further screened with the second threshold of degree $\geq 28$, closeness $\geq 0.532$ and

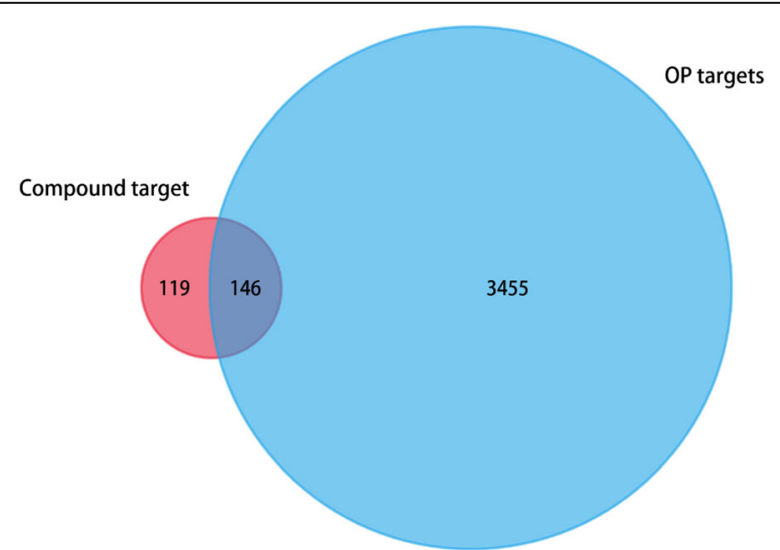

Fig. 3 The venn diagram for compound and OP targets. The overlap targets mean the potential therapeutic gene for XLGB when treating $\mathrm{OP}$ betweenness $\geq 0.004$, and 36 nodes and 439 edges remained (Fig. 4). Among these nodes, VEGFA, TP53, EGF, EGFR, PTGS2, MMP9, JUN, MAPK8, IL1B, ESR1, CAT, HSP90AA1, NOS3, MMP2, PPARG, APP, MTOR, MAPK14, NR3C1, MPO, BCL2L1, AR, IL2, KDR, VCAM1, SOD1, CYP3A4, IFNG, HIF1A, NFKB1, F2, MAP 2 K1, PGR, JAK2, COMT and MMP1 were identified (Table 1). After sorting these 36 major hub nodes and 110 other nodes in descending order, we found that VEGFA (degree $=76)$, TP53 $($ degree $=74)$, EGF $($ degree $=$ 69), EGFR (degree $=65$ ), MMP9 (degree $=64)$, PTGS2 $($ degree $=64)$, JUN $($ degree $=61)$ and MAPK8 $($ degree $=$ 60) were the key targets in this network (Fig. 5).

Then, we further built up the major hub nodecompound network (Fig. 6) based on these 36 key targets. This network included 27 compound nodes and 36 major hub target nodes. Subsequently, we reordered these compound nodes in descending order and found that quercetin was related to 17 major hub genes, luteolin to 12 genes, kaempferol to 11 genes, anhydroicaritin to 8 genes and diosgenin to 7 genes.

\section{GO biological process enrichment analysis}

After sorting 343 Biological processes (BP) terms in ascending order of $\log \mathrm{P}$ value, we found that the top 


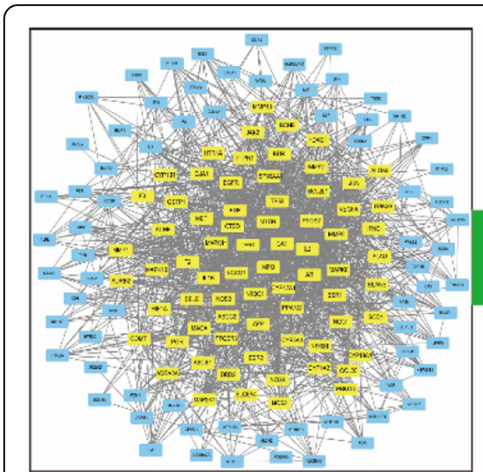

146 nodes and 1522 edges

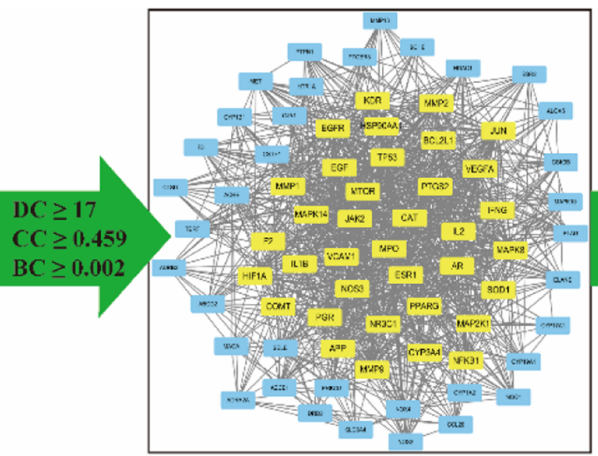

72 nodes and 942 edges

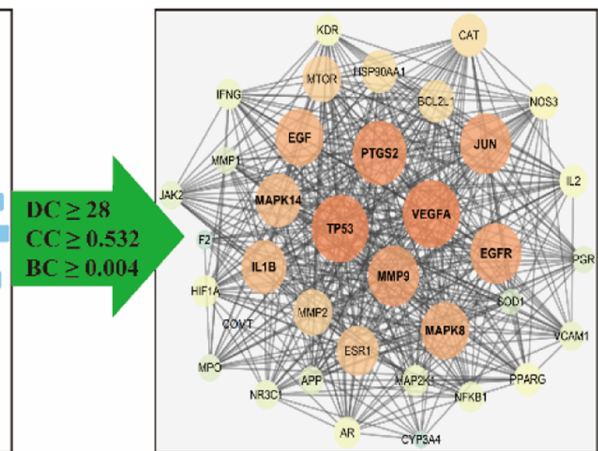

36 nodes and 439 edges

Fig. 4 The whole screening process for the PPI network through a topological method. In the third image, the bigger size and brighter color represent higher $\mathrm{DC}$ value

25 biological processes (Fig. 7) were mainly concentrated in the categories reactive oxygen species metabolic process, cellular response to organonitrogen compound and positive regulation of cell migration. Regarding the positive regulation of cell migration, the categories were positive regulation of cell migration (GO: 0030335), positive regulation of cell motility (GO: 2000147), positive regulation of cellular component movement (GO: 0051272), positive regulation of locomotion (GO: 0040017), positive regulation of MAPK cascade (GO: 0043410), and positive regulation of kinase activity (GO: 0033674). For the reactive oxygen species metabolic process, we mainly found reactive oxygen species metabolic process (GO:0072593), neurotransmitter metabolic process (GO:0042133), regulation of neurotransmitter levels (GO:0001505) and positive regulation of reactive oxygen species metabolic process (GO:2000379). Regarding the aspects of cellular response to organonitrogen compounds, we found cellular response to organonitrogen compounds (GO:0071417), cellular response to nitrogen compounds (GO:1901699) and response to peptides (GO:1901652). The remaining processes included cellular response to organic cyclic compound (GO:0071407), cellular response to hormone stimulus (GO:0032870), cellular response to lipid (GO: 0071396), circulatory system process (GO:0003013), blood circulation (GO:0008015), cytokine-mediated signaling pathway (GO:0019221), regulation of hormone levels (GO:0010817), response to inorganic substance (GO:0010035), response to lipopolysaccharide (GO:0032496), response to molecule of bacterial origin (GO:0002237), response to nutrient levels (GO: 0031667), and response to extracellular stimulus (GO: 0009991). Based on these BP enrichment analyses, we found that the anti-OP effect of XLGB may result from its regulatory effect on reactive oxygen species, organonitrogen compounds and cell migration.

\section{KEGG pathway enrichment analysis}

We further conducted KEGG pathway enrichment of 146 overlapping genes to determine the potential therapeutic mechanism of the XLGB capsules for OP. Then, we sorted 15 pathways based on the $\log \mathrm{P}$ value; these pathways included pathways in cancer (hsa05200), fluid shear stress and atherosclerosis (hsa05418), AGE-RAGE signaling pathway in diabetic complications (hsa04933), Th17 cell differentiation (hsa04659), PI3K-Akt signaling pathway (hsa04151), IL-17 signaling pathway (hsa04657), prostate cancer (hsa05215), endocrine resistance (hsa01522), HIF-1 signaling pathway (hsa04066), EGFR tyrosine kinase inhibitor resistance (hsa01521), TNF signaling pathway (hsa04668), sphingolipid signaling pathway (hsa04071), estrogen signaling pathway (hsa04915), neurotrophin signaling pathway (hsa04722) and pancreatic cancer (hsa05212) (Table 2). We also constructed a target-pathway network based on the XLGB targets enriched in each pathway (Fig. 8).

\section{Discussion}

XLGB capsules were shown to alleviate symptoms in osteoporosis patients and reverse bone loss in Ovarietomized (OVX) mice, which may result from the interaction between its active compounds and related osteoporosis targets. Thus, we investigated this potential mechanism through a network pharmacology method.

Based on the major hub gene-compound network (Fig. 6), we found that several key compounds, including quercetin, luteolin, kaempferol, anhydroicaritin, and diosgenin, play dominant roles in this network. Through a literature search, quercetin was shown to improve the bone mass and biomechanical properties of OVX rats, which is closely related to its protective effect against TNF- $\alpha$-induced impairments in bone marrow mesenchymal stem cells [35]. Similarly, luteolin was shown to increase bone formation in glucocorticoid-induced osteoporosis by reducing the excess production of 
Table 1 Information of 36 hub targets

\begin{tabular}{|c|c|c|}
\hline Uniprot ID & Gene Symbol & Description \\
\hline P15692 & VEGFA & vascular endothelial growth factor $\mathrm{A}$ \\
\hline P04637 & TP53 & tumor protein p53 \\
\hline P01133 & EGF & epidermal growth factor \\
\hline P00533 & EGFR & epidermal growth factor receptor \\
\hline P35354 & PTGS2 & prostaglandin-endoperoxide synthase 2 \\
\hline P14780 & MMP9 & matrix metallopeptidase 9 \\
\hline P05412 & $J U N$ & Jun proto-oncogene, AP-1 transcription factor subunit \\
\hline P45983 & MAPK8 & mitogen-activated protein kinase 8 \\
\hline P01584 & IL1B & interleukin 1 beta \\
\hline P03372 & ESR1 & estrogen receptor 1 \\
\hline P04040 & CAT & catalase \\
\hline P07900 & HSP90AA1 & heat shock protein 90 alpha family class A member 1 \\
\hline P29474 & NOS3 & nitric oxide synthase 3 \\
\hline P08253 & MMP2 & matrix metallopeptidase 2 \\
\hline P37231 & PPARG & peroxisome proliferator activated receptor gamma \\
\hline P05067 & APP & amyloid beta precursor protein \\
\hline P42345 & MTOR & mechanistic target of rapamycin kinase \\
\hline Q16539 & MAPK14 & mitogen-activated protein kinase 14 \\
\hline P04150 & $\mathrm{NR3C1}$ & nuclear receptor subfamily 3 group $\mathrm{C}$ member 1 \\
\hline P05164 & MPO & myeloperoxidase \\
\hline Q07817 & $\mathrm{BCL} 2 \mathrm{~L} 1$ & BCL2 like 1 \\
\hline P10275 & $A R$ & androgen receptor \\
\hline P60568 & IL2 & interleukin 2 \\
\hline P35968 & $\mathrm{KDR}$ & kinase insert domain receptor \\
\hline P19320 & VCAM1 & vascular cell adhesion molecule 1 \\
\hline P00441 & SOD1 & superoxide dismutase 1 \\
\hline P08684 & CYP3A4 & cytochrome P450 family 3 subfamily A member 4 \\
\hline P01579 & IFNG & interferon gamma \\
\hline Q16665 & HIF1A & hypoxia inducible factor 1 subunit alpha \\
\hline P19838 & NFKB1 & nuclear factor kappa B subunit 1 \\
\hline P00734 & $\mathrm{F} 2$ & coagulation factor II, thrombin \\
\hline Q02750 & MAP $2 \mathrm{~K} 1$ & mitogen-activated protein kinase kinase 1 \\
\hline P06401 & PGR & progesterone receptor \\
\hline O60674 & JAK2 & Janus kinase 2 \\
\hline P21964 & COMT & catechol-O-methyltransferase \\
\hline P03956 & MMP1 & matrix metallopeptidase 1 \\
\hline
\end{tabular}

reactive oxygen species as well as promoting the proliferation and differentiation of osteoblasts [36]. Kaempferol has also been found to prevent osteoporosis-induced bone loss in vivo and in vitro, which results from its regulatory effect on the mTOR pathway [37]. Anhydroicaritin also has an inhibitory effect on RANKL-induced osteoclast differentiation, which leads to improved bone loss in osteoporosis caused by diabetes mellitus [38]. Similar to the above compounds, diosgenin can improve the bone mineral density of OVX rats through depressing the expression of RANKL and promoting OPG [39]. These active compounds may lay the foundation for the promising anti-osteoporosis effect of XLGB capsules.

On the basis of the analysis of the target-pathway network (Fig. 8) as well as BP enrichment (Fig. 7), we speculate that the potential mechanism of XLGB is probably related to its regulation of several biological 


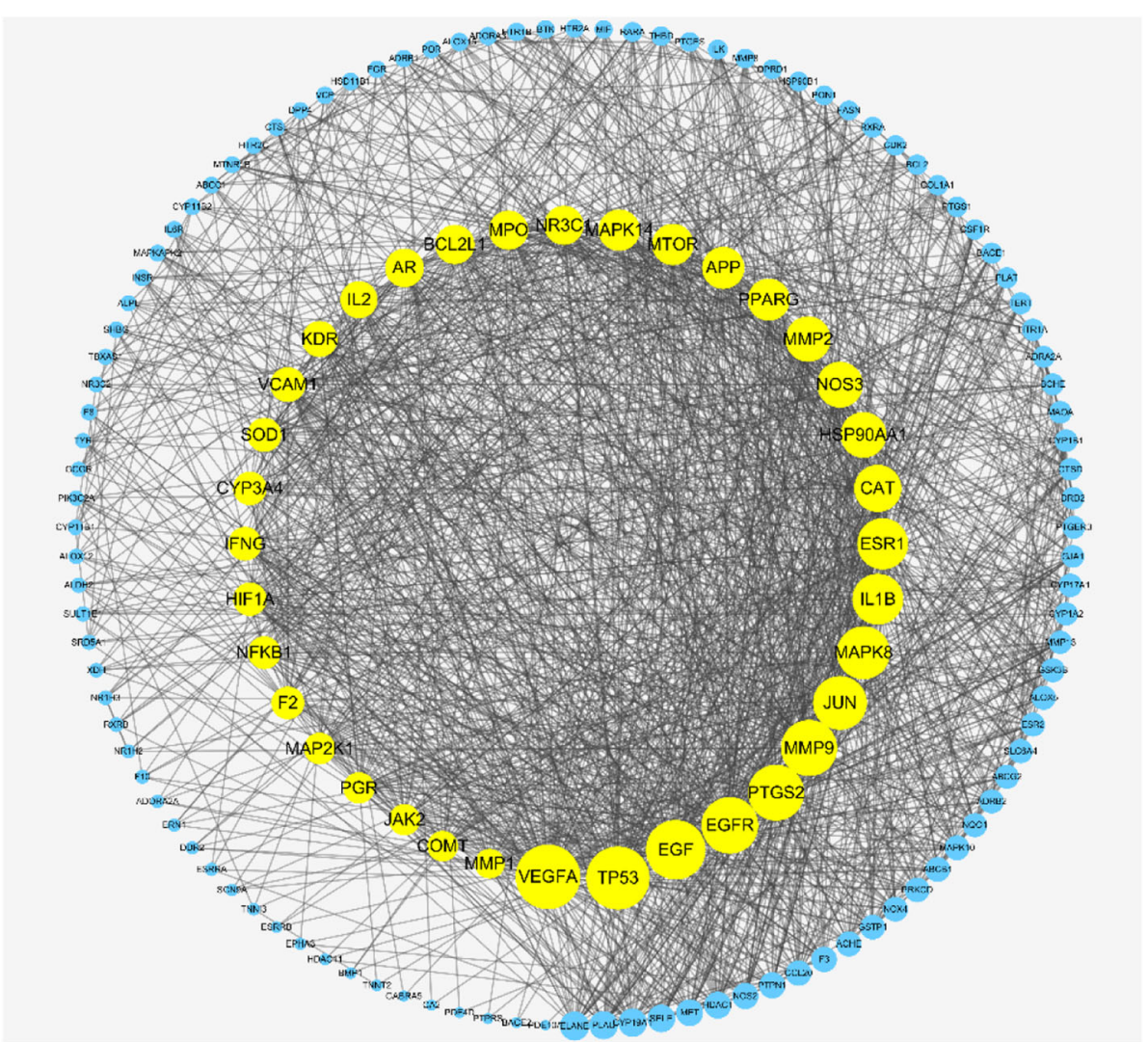

Fig. 5 The whole PPI network for 146 nodes that yellow nodes represent 36 big hub genes. All the nodes ordered by size which depended on DC value

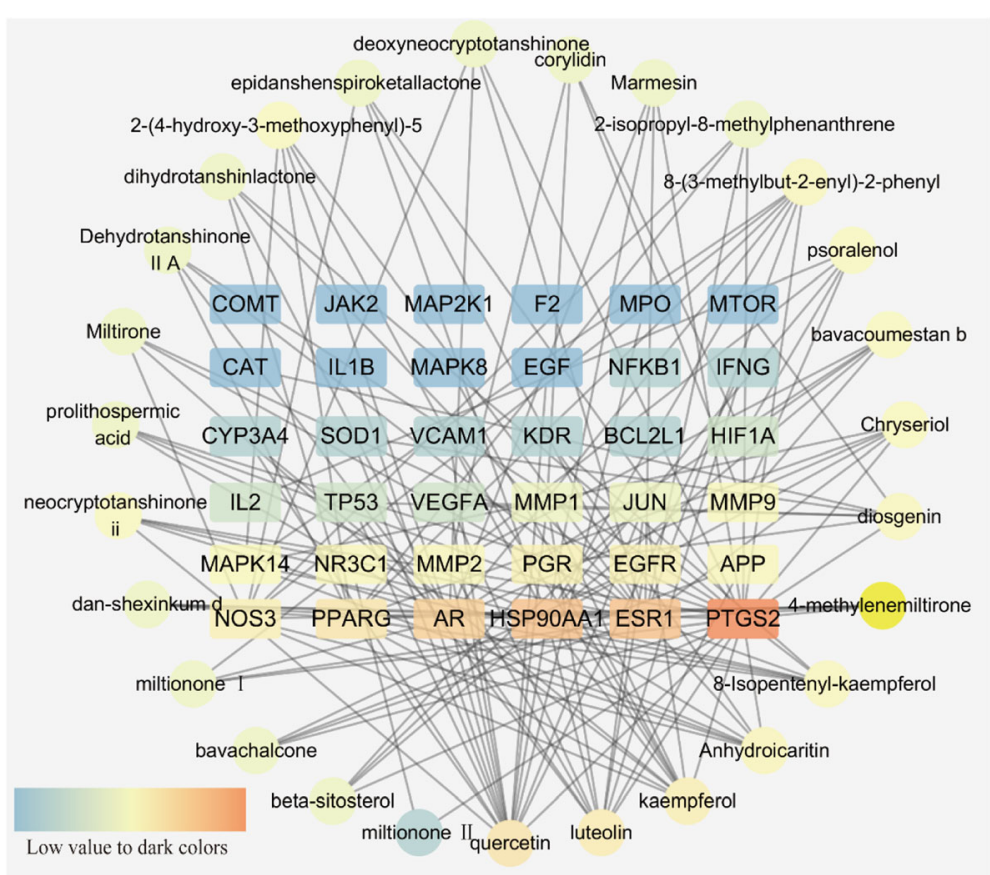

Fig. 6 The network for big hub genes-comounds connection. The square nodes are the hub genes and round ones represent compounds. And all nodes' color changes according to their degree value 


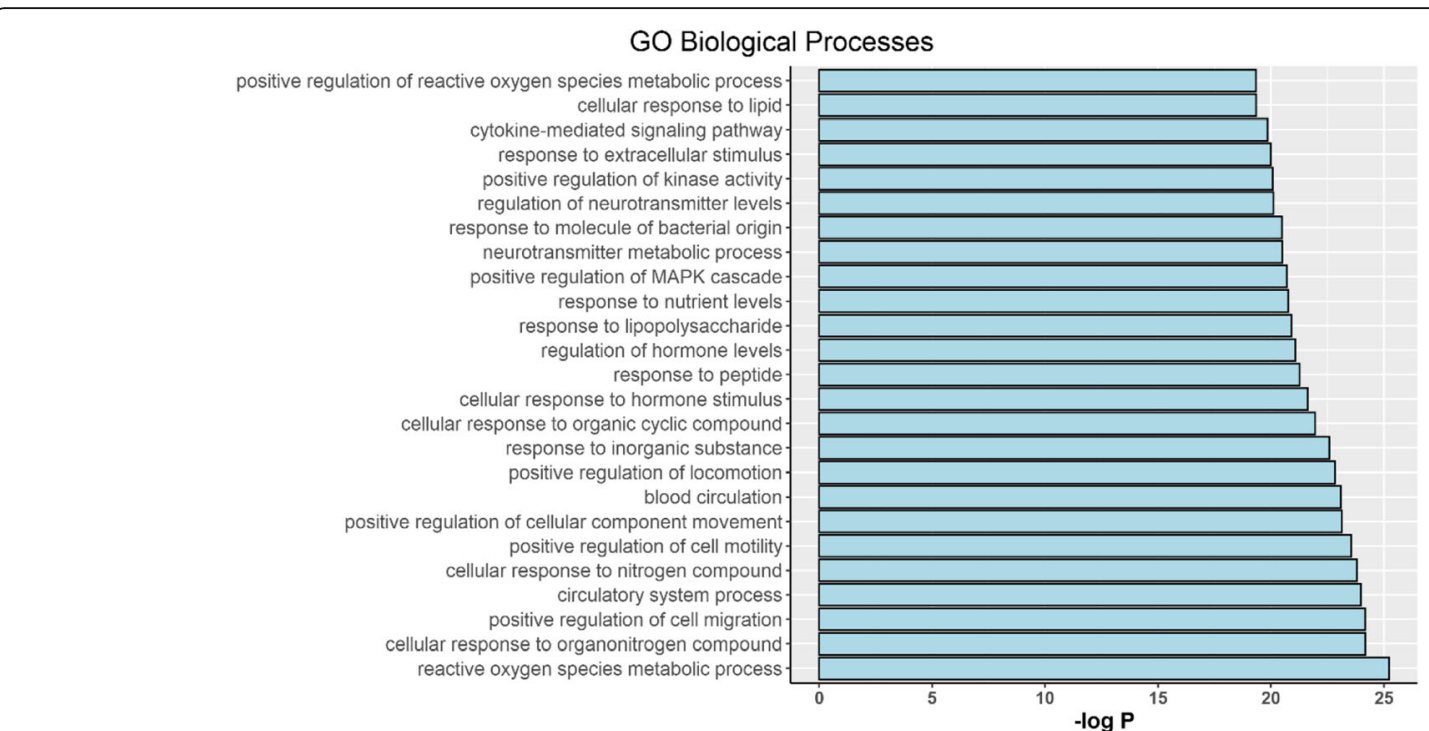

Fig. 7 Biological processes enrichment for the top 25 processe

processes, including reactive oxygen species, organonitrogen compounds and cell migration.

\section{Reactive oxygen species}

Osteoporosis is a skeletal disease that primarily results from excessive osteoclast activity or deficient osteogenesis. Reactive oxygen species (ROS), produced during aerobic respiration, have been found to play a critical role in regulating cell proliferation, apoptosis, migration and differentiation [40], as well as in the regulation of osteoclasts and osteoblasts [41, 42]. EGFR and MTOR are overlapping genes between the reactive oxygen species and the PI3K-Akt signaling pathways based on the results of BP and KEGG pathway enrichment (Fig. 9). The PI3K-Akt-mTOR signaling pathway was first found in tumor cells and was shown to regulate numerous cell functions. MTOR, a downstream molecule of Akt, controls the metabolism of sugar and fat, as well as mRNA translocation to promote protein expression. This pathway has been proven to be related to the promotion of osteogenesis through upregulating the proliferation and differentiation of bone marrow mesenchymal stem cells [43-45]. Furthermore, Jin et al. demonstrated that the alleviation of the impaired PI3K/AKT signaling pathway induced by ROS was linked to its effect against osteoporosis through reducing $\mathrm{H} 2 \mathrm{O} 2$-induced cell death in

Table 2 Information of 15 pathways

\begin{tabular}{lll}
\hline Term ID & Description & LogP \\
\hline hsa05200 & Pathways in cancer & -26.20 \\
hsa05418 & Fluid shear stress and atherosclerosis & -25.80 \\
hsa04933 & AGE-RAGE signaling pathway in diabetic complications & -19.57 \\
hsa04659 & Th17 cell differentiation & -17.43 \\
hsa04151 & Pl3K-Akt signaling pathway & -16.96 \\
hsa04657 & IL-17 signaling pathway & -16.88 \\
hsa05215 & Prostate cancer & -14.23 \\
hsa01522 & Endocrine resistance & -13.66 \\
hsa04066 & HIF-1 signaling pathway & -13.36 \\
hsa01521 & EGFR tyrosine kinase inhibitor resistance & -13.26 \\
hsa04668 & TNF signaling pathway & -11.59 \\
hsa04071 & Sphingolipid signaling pathway & -11.13 \\
hsa04915 & Estrogen signaling pathway & -10.72 \\
hsa04722 & Neurotrophin signaling pathway & -9.79 \\
hsa05212 & Pancreatic cancer & -9.76 \\
\hline
\end{tabular}




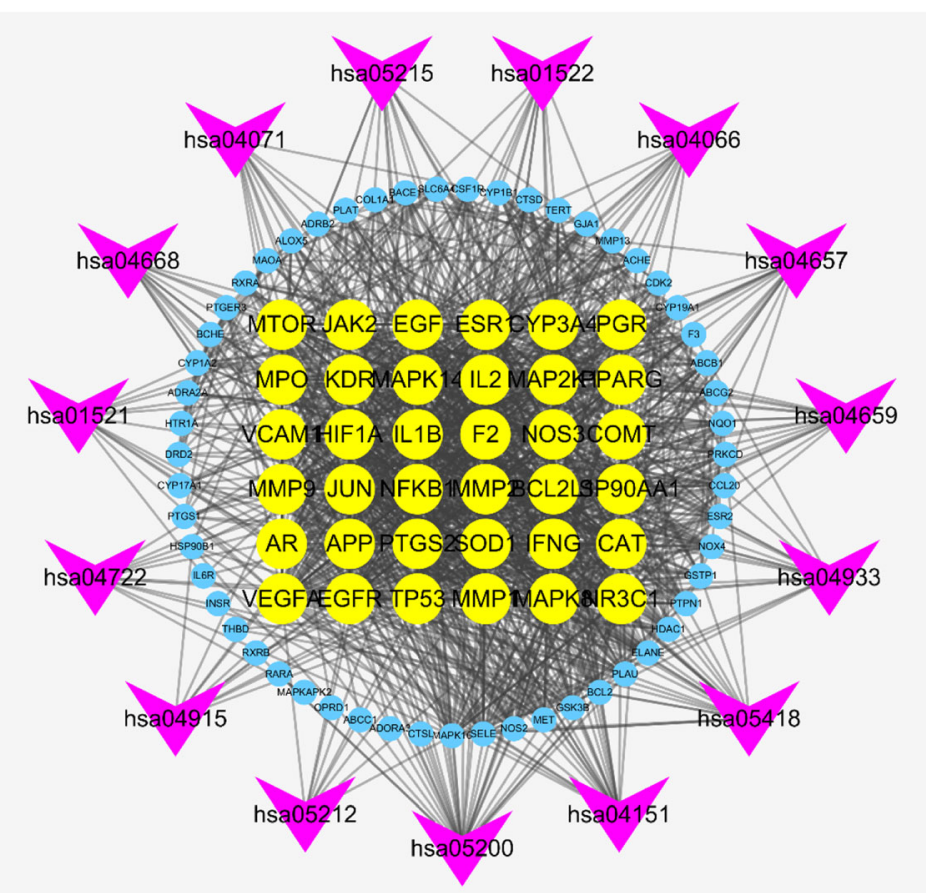

Fig. 8 The targets-pathway network of XLFB for treating OP. The circle node represent genes and the yellow ones are the big hub genes. The arrow-like nodes represent the top 15 pathways

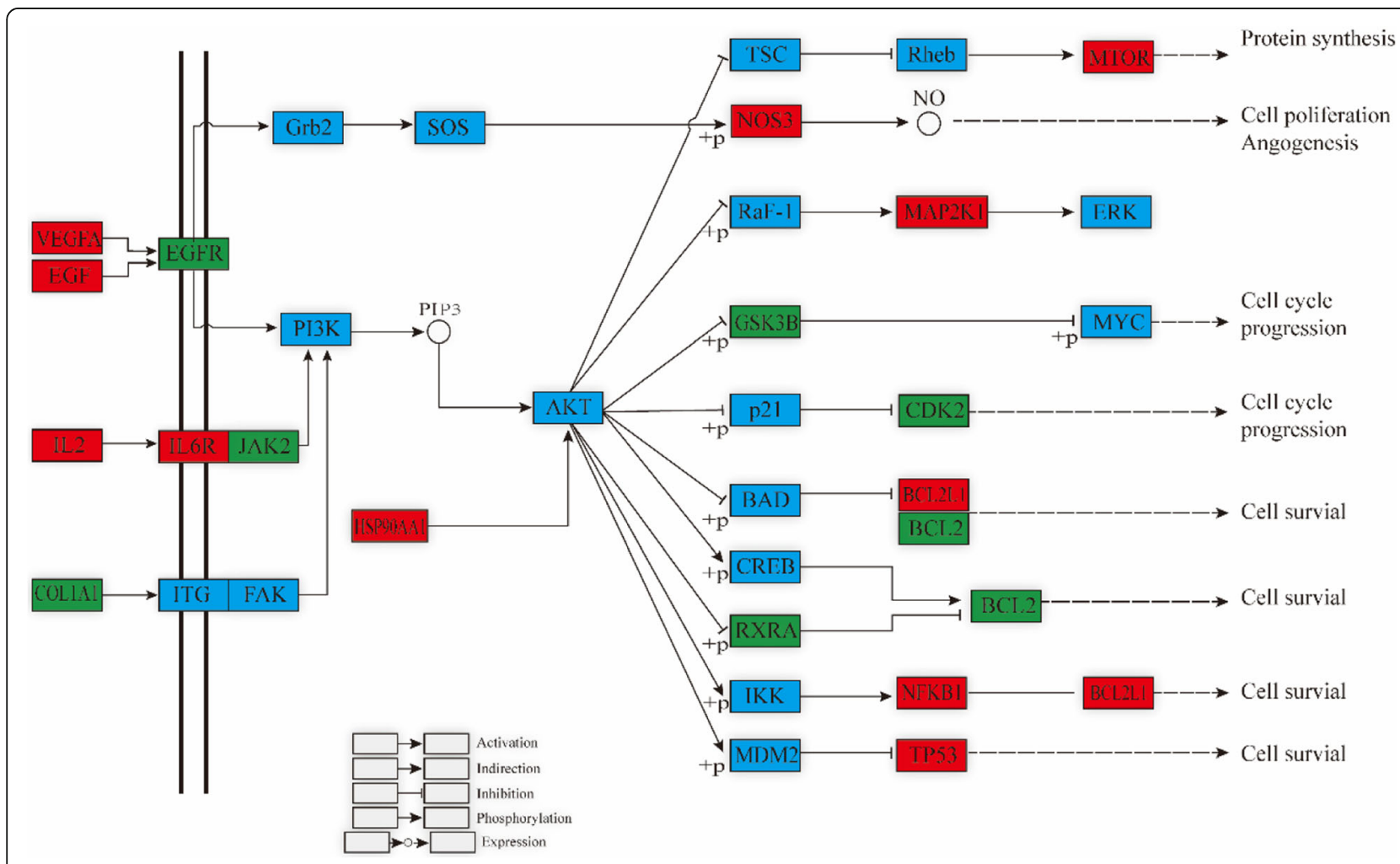

Fig. 9 PI3K-Akt signaling pathway influenced by XLGB. The red nodes represent the hub genes, the green nodes represent other genes and the blue nodes is the genes of this pathway 
MC3T3-E1 cells. Epidermal growth factor (EGF) receptor (EGFR) serves as a key regulator molecule for osteoblast functions. Previous studies have shown that the activation of the EGFR-Akt-mTOR pathway can effectively protect osteoblasts against dexamethasone, which increases the ROS production in osteoblastic cells [46].

MAPK14 is a common gene in reactive oxygen species pathways, the PI3K-Akt signaling pathway and the TNF signaling pathway. MAPKs have important roles in cell proliferation, differentiation and apoptosis, as well as in regulating inflammation [47]. ROS can lead to serious periodontal tissue destruction by promoting osteoclastic bone resorption, which is closely related to the protein expression of MAPKs and NF-kB [48]. Srinivasa et al. found that the ROS-mediated facilitation of osteoclast differentiation and resorption can be reversed through inhibiting the NF- $\mathrm{kB}$ and calcineurin-NFAT pathways [49]. The activation of MAPKs also plays a key role in osteoclastogenesis induced by RANKL. However, this ROS-directed upregulation of MAPK and NF- $\mathrm{BB}$ signaling can be attenuated through strengthening nuclear factor-erythroid 2-related factor 2 [50].

Therefore, XLGB capsule's regulatory effect on ROS is possibly due to the regulatory function of luteolin, diosgenin and anhydroicaritin. For example, luteolin, originally originating from Epimedii herba and Radix Salviae, may promote the expression of EGFR and then activate the EGFR-Akt-mTOR pathway to improve osteoblast functions. Similarly, diosgenin may also negatively regulate MTOR and subsequently activate the PI3K-AktmTOR signaling pathway to improve the osteoblastic differentiation of bone marrow mesenchymal stem cells. Moreover, anhydroicaritin may have an adverse effect on MAPK14, which further inhibits the PI3K-Akt signaling pathway and ultimately alleviates osteoporosis symptoms.

\section{Positive regulation of cell migration}

Bone mass maintenance depends upon bone formation, which is mediated by mesenchyma stem cell (MSC)-derived osteoblasts. However, the imbalance of bone formation and resorption induced by osteoblasts and osteoclasts, respectively, will cause bone diseases such as osteoporosis. MSC migration is the early stage of bone formation, as MSCs differentiate into osteoblasts after they transmigrate to the bone surface [51]. Therefore, abnormal MSC migration will influence bone homeostasis. Previous studies have found that the migration of MSCs extracted from osteoporosis patients decreased dramatically, and it was also reduced significantly in aged and OVX rats $[52,53]$. In general, insufficient migration of MSCs may be one of the vital mechanisms underlying osteoporosis, which is possibly a breakthrough for osteoporosis treatment.
EGF and EGFR were the overlapping targets between the cell migration biological process and PI3K-Akt signaling pathway. EGF and EGF-like ligands are effective chemoattractants for mesenchymal progenitors as well as for some epithelial cells, which are based on the interaction with the EGF receptor (EGFR) to promote the Akt pathway and ultimately increase MSC migration [54]. Furthermore, IL-1 $\beta$ and CCL20 are enriched in the IL-17 and TNF signaling pathways. Previous studies have demonstrated that some cytokines and chemokines, such as CCL20, can promote MSC migration. IL-1 $\beta$, produced in different tissues, also promotes the migration and adhesion of MSCs by stimulating the secretion of CCL20 and other chemokines $[55,56]$. As an important member of the matrix metalloproteinase (MMP) family, MMP9 is also enriched in the IL-17 and TNF signaling pathways. Each MMP family protease digests certain components of the extracellular matrix. In addition, MMP9 has a positive effect on promoting the migration of MSCs. Several studies have proven that upregulating MMP9 can increase MSC proliferation and migration $[57,58]$. Notably, PI3K and AKT, as important migrationpromoting factors for various cells, are related to the regulation of rBMSC migration, and the PI3K/Akt pathway contributed significantly to the increased secretion of MMP9.

In XLGB capsules, ingredients that act on these genes include luteolin, bavacoumestan B, psoralenol and bavachalcone. Based on these important components, XLGB may play a key role in regulating the migration of MSCs and then alleviate the symptoms of osteoporosis.

\section{Cellular response to organonitrogen compounds}

This cellular response to organonitrogen compounds is related to the cell state or activity change after stimulation with a compound containing at least one carbon-nitrogen bond. Bisphosphonates (BPs) are the most frequently used treatments for osteoporosis to inhibit excessive osteoclast resorption activity. There are two different kinds of BPs that restrain osteoclast resorption. Non-nitrogencontaining BPs induce osteoclast apoptosis by inhibiting adenosine triphosphate-dependent enzymes. However, nitrogen-containing bisphosphonates (N-BPs) suppress the activation of farnesyl diphosphate synthase, which is necessary for osteoclast differentiation [59]. Notably, BPs containing nitrogen increased the inhibitory potency by 10-10,000 times compared to non-nitrogen-containing BPs [60]. In addition, N-BPs also inhibit preosteoclasts by inhibiting the PI3K/Akt pathway [61].

In the case of this organonitrogen compound response process, NF-kB1 is enriched in the PI3K-Akt, TNF, TH17, IL17, HIF and fluid shear signaling pathways. NF$\kappa \mathrm{B}$ is a critical transcription factor composed of Rel proteins, NF- $\kappa B 1$ and NF- $\kappa B 2$. NF- $\kappa B$ signaling activation is 
one of the downstream events after the stimulation of RANKL, which induces the differentiation and bone resorption of osteoclasts [62]. The XLGB formula may exert its anti-osteoporosis effect through regulating the cell response towards organonitrogen compounds and then inhibiting the expression level of NF- $\mathrm{kB}$, eventually reducing the differentiation and bone resorption of osteoclasts.

\section{Conclusion}

By utilizing network pharmacology, we explored the potential targets of XLGB compounds and investigated the underlying mechanism of their anti-osteoporosis effects, which may be based on three key biological processes: the inhibition of reactive oxygen species, promotion of the organonitrogen compound response and cell migration. According to the KEGG pathway enrichment results, we found that the PI3K-Akt signaling pathway is the main pathway when XLGB capsules are used to treat osteoporosis. Thus, we believe that the anti-osteoporotic effect of XLGB is mainly based on its direct or indirect regulation of the above potential targets and pathways, and our results provide promising directions for future research, which is essential to reveal the exact regulatory mechanisms.

\begin{abstract}
Abbreviations
BP: Biological processes; DL: Drug-likeness; GO: Gene ontology; KEGG: Kyoto encyclopedia of genes and genomes; OB: Oral bioavailability;

OP: Osteoporosis; TCM: Traditional Chinese medicine; OVX: Ovarietomized; ROS: Reactive oxygen species; MSC: Mesenchyma stem cell; YYH: Epimedium sagittatum maxim; DS: Salvia miltiorrhiza bunge; DH: Rehmannia glutinosa steud; XD: Dipsacus inermis wall; ZM: Anemarrhena asphodeloides bunge; BGZ: Psoralea corylifolia L.
\end{abstract}

\section{Acknowledgements}

Not applicable.

\section{Authors' contributions}

$\mathrm{HSB}$ and $\mathrm{XL}$ conceived the idea of this article and supervised the research. HSB performed the research, analyzed the data and wrote the manuscript. HZG and ZQF performed target prediction and analysis as well as related enrichment processes. $\mathrm{XL}$ participated in revising the data and improving the manuscript. All authors reviewed the manuscript. All authors read and approved the final version of the manuscript

\section{Funding}

None.

\section{Availability of data and materials \\ All major data used to support the findings of this study have been deposited in the figshare repository (https://doi.org/10.6084/m9.figshare. 11341358.v1). The datasets supporting the conclusions of this article are available in public databases from TCMSP, TCMID, Swiss Target Prediction, GeneCards, CTD, TTD, and PharmGKB.}

\section{Ethics approval and consent to participate}

Not applicable.

\section{Consent for publication}

Not applicable.

\section{Competing interests}

The authors declare that they have no competing interests.

\section{Author details}

${ }^{1}$ Foshan Hospital of Traditional Chinese Medicine, Foshan 528000, China. ${ }^{2}$ First Clinical Medical College, Guangzhou University of Chinese Medicine, Guangzhou 510405, China. ${ }^{3}$ Department of Nephrology, The First Affiliated Hospital of Guangzhou University of Chinese Medicine, Guangzhou 510405, China.

Received: 10 December 2019 Accepted: 26 June 2020

Published online: 03 July 2020

\section{References}

1. Chen K, Qiu P, Yuan Y, Zheng L, He J, Wang C, Guo Q, Kenny J, Liu Q, Zhao J. Pseurotin a inhibits Osteoclastogenesis and prevents Ovariectomizedinduced bone loss by suppressing reactive oxygen species. Theranostics. 2019;9(6):1634.

2. Lewiecki EM, Ortendahl JD, Vanderpuye-Orgle J, Grauer A, Arellano J, Lemay J, Harmon AL, Broder MS, Singer AJ. Healthcare policy changes in osteoporosis can improve outcomes and reduce costs in the United States. JBMR plus. 2019;3(9):e10192.

3. Letarouilly J-G, Broux O, Clabaut A. New insights into the epigenetics of osteoporosis. Genomics. 2019;111(4):793-8.

4. Einhorn TA, Bogdan Y, Tornetta P III. Bisphosphonate-associated fractures of the femur: pathophysiology and treatment. J Orthop Trauma. 2014;28(7): 433-8.

5. Tabatabaei-Malazy O, Salari P, Khashayar P, Larijani B. New horizons in treatment of osteoporosis. DARU J Pharm Sci. 2017;25(1):2.

6. Xiao $L$, Tao R. Traditional Chinese medicine (TCM) therapy. Adv Exp Med Biol. 2017;1010:261-80

7. Xu W, Towers A, Li P, COLLET JP. Traditional Chinese medicine in cancer care: perspectives and experiences of patients and professionals in China. Eur J Cancer Care. 2006;15(4):397-403.

8. Sham T-T, Chan C-O, Wang Y-H, Yang J-M, Mok DK-W, Chan S-W. A review on the traditional Chinese medicinal herbs and formulae with hypolipidemic effect. Biomed Res Int. 2014;2014.

9. Ping L. YANG L-p, GONG Y-w: application of systems biology Technology in Research of traditional Chinese medicine. J Tradit Chin Med. 2009;29(2):1537.

10. Zhou LP, Wong KY, Yeung HT, Dong XL, Xiao HH, Gong AG, Tsim KW, Wong MS. Bone protective effects of Danggui Buxue Tang alone and in combination with Tamoxifen or Raloxifene in vivo and in vitro. Front Pharmacol. 2018:9:779.

11. Gu F, Jiang J, Wang S, Feng T, Zhou Y, Ma Y, Shen S. An experimental research into the potential therapeutic effects of anti-osteoporosis decoction and Yougui pill on ovariectomy-induced osteoporosis. Am J Transl Res. 2019;11(9):6032-9.

12. Wang X, He Y, Guo B, Tsang M-C, Tu F, Dai Y, Yao Z, Zheng L, Xie X, Wang $\mathrm{N}$. In vivo screening for anti-osteoporotic fraction from extract of herbal formula Xianlinggubao in ovariectomized mice. PLoS One. 2015;10(2): e0118184.

13. Hu C. Cheng M: clinical observation on 30 casesof Xianlinggubao capsules in treatement of osteoporosis. Chin Tradit Pat Med. 2000;22:246-7.

14. Zhu H, Qin L, Garnero P, Genant H, Zhang G, Dai K, Yao X, Gu G, Hao Y, Li Z. The first multicenter and randomized clinical trial of herbal Fufang for treatment of postmenopausal osteoporosis. Osteoporos Int. 2012;23(4): 1317-27.

15. Zhang W, Huai Y, Miao Z, Qian A, Wang Y. Systems pharmacology for investigation of the mechanisms of action of traditional Chinese medicine in drug discovery. Front Pharmacol. 2019;10:743.

16. Wang N, Xu P, Wang X, Yao W, Yu Z, Wu R, Huang X, Si Y, Shou D. Integrated pathological cell fishing and network pharmacology approach to investigate main active components of Er-Xian decotion for treating osteoporosis. J Ethnopharmacol. 2019;241:111977.

17. Zhang W, Xue K, Gao Y, Huai Y, Wang W, Miao Z, Dang K, Jiang S, Qian A. Systems pharmacology dissection of action mechanisms of Dipsaci Radix for osteoporosis. Life Sci. 2019;235:116820.

18. Ru J, Li P, Wang J, Zhou W, Li B, Huang C, Li P, Guo Z, Tao W, Yang Y. TCMSP: a database of systems pharmacology for drug discovery from herbal medicines. J Cheminformatics. 2014;6(1):13. 
19. Xue R, Fang Z, Zhang M, Yi Z, Wen C, Shi T. TCMID: traditional Chinese medicine integrative database for herb molecular mechanism analysis. Nucleic Acids Res. 2012;41(D1):D1089-95.

20. Xu X, Zhang W, Huang C, Li Y, Yu H, Wang Y, Duan J, Ling Y. A novel chemometric method for the prediction of human oral bioavailability. Int $J$ Mol Sci. 2012;13(6):6964-82.

21. Tao W, Xu X, Wang X, Li B, Wang Y, Li Y, Yang L. Network pharmacologybased prediction of the active ingredients and potential targets of Chinese herbal Radix Curcumae formula for application to cardiovascular disease. J Ethnopharmacol. 2013;145(1):1-10.

22. Daina A, Michielin O, Zoete $V$. SwissADME: a free web tool to evaluate pharmacokinetics, drug-likeness and medicinal chemistry friendliness of small molecules. Sci Rep. 2017;7:42717.

23. Grondin CJ, Davis AP, Wiegers TC, Wiegers JA, Mattingly CJ. Accessing an expanded exposure science module at the comparative Toxicogenomics database. Environ Health Perspect. 2018;126(1):014501.

24. Rebhan M, Chalifa-Caspi V, Prilusky J, Lancet D. GeneCards: a novel functional genomics compendium with automated data mining and query reformulation support. Bioinformatics. 1998;14(8):656-64.

25. Li YH, Yu CY, Li XX, Zhang P, Tang J, Yang Q, Fu T, Zhang X, Cui X, Tu G. Therapeutic target database update 2018: enriched resource for facilitating bench-to-clinic research of targeted therapeutics. Nucleic Acids Res. 2017; 46(D1):D1121-7.

26. Hamosh A, Scott AF, Amberger JS, Bocchini CA, McKusick VA. Online Mendelian Inheritance in Man (OMIM), a knowledgebase of human genes and genetic disorders. Nucleic Acids Res. 2005;33(suppl_1):D514-7.

27. Whirl-Carrillo M, McDonagh EM, Hebert J, Gong L, Sangkuhl K, Thorn C, Altman RB, Klein TE. Pharmacogenomics knowledge for personalized medicine. Clin Pharmacol Ther. 2012;92(4):414-7.

28. Szklarczyk D, Morris JH, Cook H, Kuhn M, Wyder S, Simonovic M, Santos A, Doncheva NT, Roth A, Bork P. The STRING database in 2017: qualitycontrolled protein-protein association networks, made broadly accessible. Nucleic Acids Res. 2016;45:362-8

29. Shannon P, Markiel A, Ozier O, Baliga NS, Wang JT, Ramage D, Amin N, Schwikowski B, Ideker T. Cytoscape: a software environment for integrated models of biomolecular interaction networks. Genome Res. 2003;13(11): 2498-504

30. Missiuro PV, Liu K, Zou L, Ross BC, Zhao G, Liu JS, Ge H. Information flow analysis of interactome networks. PLoS Comput Biol. 2009;5(4):e1000350.

31. Raman K, Damaraju N, Joshi GK. The organisational structure of protein networks: revisiting the centrality-lethality hypothesis. Syst Synth Biol. 2014; $8(1): 73-81$.

32. Tang Y, Li M, Wang J, Pan Y, Wu F-X. CytoNCA: a cytoscape plugin for centrality analysis and evaluation of protein interaction networks. BioSyst. 2015;127:67-72

33. Zhou Y, Zhou B, Pache L, Chang M, Khodabakhshi AH, Tanaseichuk O, Benner C, Chanda SK. Metascape provides a biologist-oriented resource for the analysis of systems-level datasets. Nat Commun. 2019:10(1):1523.

34. Chen L, Zhang Y-H, Wang S, Zhang Y, Huang T, Cai Y-D. Prediction and analysis of essential genes using the enrichments of gene ontology and KEGG pathways. PLoS One. 2017;12(9):e0184129.

35. Yuan Z, Min J, Zhao Y, Cheng Q, Wang K, Lin S, Luo J, Liu H. Quercetin rescued TNF-alpha-induced impairments in bone marrow-derived mesenchymal stem cell osteogenesis and improved osteoporosis in rats. Am J Transl Res. 2018;10(12):4313-21.

36. Jing Z, Wang C, Yang Q, Wei X, Jin Y, Meng Q, Liu Q, Liu Z, Ma X, Liu K, et al. Luteolin attenuates glucocorticoid-induced osteoporosis by regulating ERK/Lrp-5/GSK-3beta signaling pathway in vivo and in vitro. J Cell Physiol. 2019;234(4):4472-90

37. Zhao J, Wu J, Xu B, Yuan Z, Leng Y, Min J, Lan X, Luo J. Kaempferol promotes bone formation in part via the mTOR signaling pathway. Mol Med Rep. 2019;20(6):5197-207.

38. Zheng ZG, Zhang X, Zhou YP, Lu C, Thu PM, Qian C, Zhang M, Li P, Li HJ, Xu X. Anhydroicaritin, a SREBPs inhibitor, inhibits RANKL-induced osteoclastic differentiation and improves diabetic osteoporosis in STZinduced mice. Eur J Pharmacol. 2017:809:156-62.

39. Zhang Z, Song C, Fu X, Liu M, Li Y, Pan J, Liu H, Wang S, Xiang L, Xiao $G G$, et al. High-dose diosgenin reduces bone loss in ovariectomized rats via attenuation of the RANKL/OPG ratio. Int J Mol Sci. 2014;15(9): $17130-47$.
40. Domazetovic V, Marcucci G, lantomasi T, Brandi ML, Vincenzini MT. Oxidative stress in bone remodeling: role of antioxidants. Clin Cases Miner Bone Metab. 2017;14(2):209.

41. Schröder K. NADPH oxidases in bone homeostasis and osteoporosis. Free Radic Biol Med. 2019;132:67-72.

42. Gan X, Huang S, Yu Q, Yu H, Yan SS. Blockade of Drp1 rescues oxidative stress-induced osteoblast dysfunction. Biochem Biophys Res Commun. 2015; 468(4):719-25.

43. Luo G, Xu B, Huang Y. Icariside II promotes the osteogenic differentiation of canine bone marrow mesenchymal stem cells via the PI3K/AKT/mTOR/S6K1 signaling pathways. Am J Transl Res. 2017;9(5):2077.

44. Li G, Hu J, Chen H, Chen L, Zhang N, Zhao L, Wen N, Yang Y. Enamel matrix derivative enhances the proliferation and osteogenic differentiation of human periodontal ligament stem cells on the titanium implant surface. Organogenesis. 2017;13(3):103-13.

45. Tong $Y$, Feng $W$, Wu Y, Lv H, Jia Y, Jiang D. Mechano-growth factor accelerates the proliferation and osteogenic differentiation of rabbit mesenchymal stem cells through the PI3K/AKT pathway. BMC Biochem. 2015;16(1):1.

46. J-b F, Liu W, Zhu X-h, Yuan K, Xu D-w, Chen J-j, Cui Z-m. EGFR-AKT-mTOR activation mediates epiregulin-induced pleiotropic functions in cultured osteoblasts. Mol Cell Biochem. 2015;398(1-2):105-13.

47. Berghe WV, Plaisance S, Boone E, De Bosscher K, Schmitz ML, Fiers W, Haegeman G. p38 and extracellular signal-regulated kinase mitogenactivated protein kinase pathways are required for nuclear factor-kB p65 transactivation mediated by tumor necrosis factor. J Biol Chem. 1998;273(6): 3285-90.

48. An Y, Zhang H, Wang C, Jiao F, Xu H, Wang X, Luan W, Ma F, Ni L, Tang X. Activation of ROS/MAPKs/NF-KB/NLRP3 and inhibition of efferocytosis in osteoclast-mediated diabetic osteoporosis. FASEB J. 2019:33(11):12515-27.

49. Srinivasan S, Koenigstein A, Joseph J, Sun L, Kalyanaraman B, Zaidi M, Avadhani NG. Role of mitochondrial reactive oxygen species in osteoclast differentiation. Ann N Y Acad Sci. 2010;1192(1):245.

50. Thummuri D, Naidu V, Chaudhari P. Carnosic acid attenuates RANKLinduced oxidative stress and osteoclastogenesis via induction of Nrf2 and suppression of NF-KB and MAPK signalling. J Mol Med. 2017;95(10):1065-76.

51. Liu TM, Lee EH. Transcriptional regulatory cascades in Runx2-dependent bone development. Tissue Eng B Rev. 2012;19(3):254-63.

52. Haasters F, Docheva D, Gassner C, Popov C, Böcker W, Mutschler W, Schieker M, Prall WC. Mesenchymal stem cells from osteoporotic patients reveal reduced migration and invasion upon stimulation with BMP-2 or BMP-7. Biochem Biophys Res Commun. 2014;452(1):118-23.

53. Sanghani-Kerai A, Coathup M, Samazideh S, Kalia P, Silvio LD, Idowu B, Blunn G. Osteoporosis and ageing affects the migration of stem cells and this is ameliorated by transfection with CXCR4. Bone Joint Res. 2017;6(6): 358-65.

54. Zhu J, Siclari VA, Liu F, Spatz JM, Chandra A, Pajevic PD, Qin L. Amphiregulin-EGFR signaling mediates the migration of bone marrow mesenchymal progenitors toward PTH-stimulated osteoblasts and osteocytes. PLoS One. 2012;7(12):e50099.

55. Mardpour S, Hamidieh AA, Taleahmad S, Sharifzad F, Taghikhani A, Baharvand $\mathrm{H}$. Interaction between mesenchymal stromal cell-derived extracellular vesicles and immune cells by distinct protein content. J Cell Physiol. 2019;234(6):8249-58.

56. Carrero R, Cerrada I, Lledó E, Dopazo J, García-García F, Rubio M-P, Triqueros C, Dorronsoro A, Ruiz-Sauri A, Montero JA. IL1 $\beta$ induces mesenchymal stem cells migration and leucocyte chemotaxis through NF-kB. Stem Cell Rev Rep. 2012;8(3):905-16.

57. Zhang W, Tu G, Lv C, Long J, Cong L, Han Y. Matrix metalloproteinase-9 is up-regulated by CCL19/CCR7 interaction via PI3K/Akt pathway and is involved in CCL19-driven BMSCs migration. Biochem Biophys Res Commun. 2014;451(2):222-8

58. Zhang Z, Zhu L, Feng P, Tan Y, Zhang B, Gao E, Wang X, Fan C, Wang X, Yi W. C1q/tumor necrosis factor-related protein-3-engineered mesenchymal stromal cells attenuate cardiac impairment in mice with myocardial infarction. Cell Death Dis. 2019;10(7):1-15.

59. Yuan H, Niu L-n, Jiao K, Pei D-d, Pramanik C, Li J-y, Messer R, Kumar S, Pashley DH, Tay FR. Revival of nitrogen-containing bisphosphonate-induced inhibition of osteoclastogenesis and osteoclast function by water-soluble microfibrous borate glass. Acta Biomater. 2016:31:312-25. 
60. Dunford JE, Thompson K, Coxon FP, Luckman SP, Hahn FM, Poulter CD, Ebetino FH, Rogers MJ. Structure-activity relationships for inhibition of farnesyl diphosphate synthase in vitro and inhibition of bone resorption in vivo by nitrogen-containing bisphosphonates. J Pharmacol Exp Ther. 2001;296(2):235-42.

61. Tsubaki M, Komai M, Itoh T, Imano M, Sakamoto K, Shimaoka H, Takeda T, Ogawa N, Mashimo K, Fujiwara D. Nitrogen-containing bisphosphonates inhibit RANKL-and M-CSF-induced osteoclast formation through the inhibition of ERK1/2 and Akt activation. J Biomed Sci. 2014; 21(1):10.

62. Ono T, Nakashima T. Recent advances in osteoclast biology. Histochem Cell Biol. 2018;149(4):325-41.

\section{Publisher's Note}

Springer Nature remains neutral with regard to jurisdictional claims in published maps and institutional affiliations.

Ready to submit your research? Choose BMC and benefit from:

- fast, convenient online submission

- thorough peer review by experienced researchers in your field

- rapid publication on acceptance

- support for research data, including large and complex data types

- gold Open Access which fosters wider collaboration and increased citations

- maximum visibility for your research: over $100 \mathrm{M}$ website views per year

At $B M C$, research is always in progress.

Learn more biomedcentral.com/submissions 